\title{
Neue Aspekte der Desinformation
}

Die Freiheit der Beschaffung und Weitergabe von Informationen kann - auch ohne Einmischung öffentlicher Behörden - absichtlich verzerrt werden. In EU-Dokumenten wird sie in solchen Fällen sogar als eine neue "Waffe" bewertet und als Desinformation benannt. Durch die Ausbreitung des Internets kann das Recht auf Information auch dazu benutzt werden, die Grundprinzipien der Europäischen Union zu untergraben, mit dem indirekten Ziel, Diskussionen und kritisches Denken zu verhindern. Desinformation, als eine extreme Form des Medienmissbrauchs mit dem Ziel der Beeinflussung sozialer und politischer Prozesse, wird manchmal sogar von Regierungen gesponsert und in den internationalen Beziehungen eingesetzt. Beispiele hierfür sind die Desinformationskampagne zum Brexit oder die Einmischung in vergangene US-Wahlen.

\section{Desinformation als Gefahr}

Desinformation ermöglicht die Manipulation der öffentlichen Meinung und damit die Beeinflussung der Innenpolitik sowohl einzelner souveräner Staaten als auch der EU insgesamt. Insbesondere der Europäische Wirtschafts- und Sozialausschuss (EWSA) hält die Gefahren, die mit entsprechenden offensiven CyberOperationen verbunden sind, für unterschätzt. ${ }^{1}$

Die Analyse der jüngsten europäischen Dokumente zeigt deutliche Unterschiede zwischen dem EWSA und der Europäischen Kommission in ihrer Herangehensweise an Sozial-Media-Platt-

1 Stellungnahme des Europäischen Wirtschafts- und Sozialausschusses zu der Mitteilung der Kommission an das Europäische Parlament, den Rat, den Europäischen Wirtschafts- und Sozialausschuss und den Ausschuss der Regionen: Bekämpfung von Desinformation im Internet: ein europäischer Ansatz [COM(2018) 236 final]. ABI. C 440 vom 6.12.2018, S. 183, S. 186.

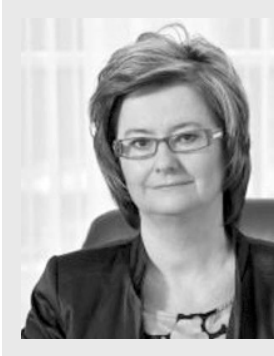

\section{Prof. Dr. habil. Irena Lipowicz}

Kardinal-Stefan-Wyszyński-Universität in Warschau, Inhaberin des Lehrstuhls für Verwaltungs- und Kommunalwesen, Dr. h.c. der Universität Osnabrück, Viadrina-Preisträgerin

E-Mail: i.lipowicz@uksw.edu.pl

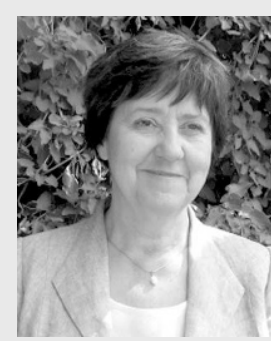

\section{Prof. Dr. habil. Grażyna Szpor}

Kardinal-Stefan-Wyszyński-Universität in Warschau, Inhaberin des Lehrstuhls für Informationsrecht

E-Mail: g.szpor@uksw.edu.pl formen; während die Kommission die Erlangung neuer Erkenntnisse durch Forschung und Strategiepapiere betont, kritisiert der EWSA das Fehlen verbindlicher, praktischer Schritte zur Bekämpfung von Desinformation. Natürlich sollte die EU ihre Forschungen zur Desinformation fortsetzen, aber der EWSA bringt nachdrücklich die Kategorie der Widerstandsfähigkeit der Europäer (EU-Bürger) gegen Desinformation in die Debatte ein, die durch das „Eurobarometer“ ständig überwacht werden sollte. Der EWSA kritisiert außerdem, dass es der Kommission ,an Ehrgeiz mangelt“, dass sie das Thema nicht mit ausreichender Dringlichkeit behandelt und dass sie nicht zum Kern der Sache vordringt. Die Schaffung eines europäischen Unterstützungssystems für Qualitätsmedien, das „das Grundrecht der Bürger auf hochwertige und zuverlässige Informationen" sichern soll, ist eine bemerkenswerte Entwicklung; nach jahrzehntelangem Kampf für das Recht auf Information und seine Varianten - z. B. das Recht auf freie Rechtsauskunft - kommen wir zu einer bedeutenden Modifikation: das Recht auf Information dient der Demokratie und dem Schutz der Freiheiten, wenn es Qualität und Zuverlässigkeit von Informationen sicherstellt. Es geht um die Sicherstellung der Transparenz von Algorithmen, insbesondere derjenigen, die Online-Systemen, einschließlich Plattformen wie Facebook, zugrunde liegen, und um die Sicherstellung einer größeren Aufsicht über diese Algorithmen. Es geht auch um die Transparenz von Algorithmen, mit denen Menschen „verwaltet“ werden, z. B. Predictive Policing. Auch hier gibt es eine deutliche Divergenz zwischen der Kommission, dem Europäischen Parlament und dem EWSA, der, wenn auch vorsichtig, nachdrücklicher als die Kommission davon spricht, „die Möglichkeit zu prüfen, Informationsmonopole aufzubrechen“ und „die Möglichkeit zu untersuchen, eine größere Transparenz und Kontrolle von Algorithmen zu gewährleisten“. 2

2 Ibid.; s. a. Informationsbericht "Nutzung der Medien zur Einflussnahme auf gesellschaftliche und politische Prozesse in der EU und ihren östlichen Nachbarländern" (REX/432); Gemeinsame Mitteilung an das Europäische Parlament, den Europäischen Rat, den Rat, den Europäischen Wirtschafts- und Sozialausschuss 
Desinformation wird derzeit auf europäischer und nationaler Ebene vor allem als - völlig neue und komplexe - Bedrohung für die Landesverteidigung und als Instrument der Beeinflussung von Wahlergebnissen durch den Einsatz fortgeschrittener und verdeckter Methoden des Micro-Targeting betrachtet. Man kann sagen, dass bei der Reflexion über die Folgen der fortgeschrittenen Desinformation für den Staat vor allem die axiologische und verfassungsrechtliche Ebene untersucht wird, die zum Auftreten einer Situation der „Demokratie unter Druck“ führt. Diese Forschung hat dazu geführt, den begrifflichen Apparat der Politikwissenschaft und des Rechts mit Konzepten wie digitale Souveränität, digitale Selbstverteidigung, Überwachungsstaat und anderen zu erweitern.

Es ist zu fragen, warum Desinformation in einem Wahlprozess heute eine so fundamentale Bedrohung für das Funktionieren des Staates ist. Fake News gab es auch vorher schon, aber erst durch den Cambrigde Analytica-Skandal wurde die Annahme, dass an Wahlen aktive, kritische, unabhängige Bürger, die von Natur aus immun gegen Manipulation sind, teilnehmen, vollständig als juristische Fiktion entlarvt. In traditionellen Wahlkämpfen gab es noch eine gewisse „Waffengleichheit“; der durchschnittliche Wähler konnte durch die Massenpropaganda getäuscht werden, konnte aber dennoch ein gewisses eigenständiges Denken an den Tag legen, obwohl dies auch damals schon eine idealistische Annahme war. Der rasante Fortschritt der Sozialwissenschaften in diesem eigentümlichen Wettrüsten führt dazu, dass der Durchschnittsbürger mit Desinformation und Manipulation konfrontiert wird, die auf Wissen basieren: Psychologie, einschließlich Sozialpsychologie, Soziologie, Informationstheorie, Informatik und Systemtheorie. Die Erfindung des Mikroprofils, das dank der zuvor geschaffenen Informationsbasis alle Zweifel und Fragen vorwegnimmt und nicht nur die Argumentation, sondern, schlimmer noch, die Informationsbasis des Entscheidungsprozesses eines Individuums individualisiert, macht den Rezipienten, selbst einen aktiven, unabhängigen und intelligenten Wähler, praktisch wehrlos. Plattformen und soziale Medien modifizieren die bereitgestellten Informationen, verstärken den Trend früherer Recherchen und schließen den Bürger und Wähler in einer luftdichten Informationsblase ein, während die Informationen, die er besitzt, viele seiner Entscheidungen bestimmen, nicht nur die Wahlentscheidungen.

\section{Maßnahmen gegen Desinformation}

Zu den konkreten Maßnahmen zur Bekämpfung von Desinformation, die eher die Ausnahme als die Regel sind, zählt z. B. die 2015 von der Kommission ins Leben gerufene Task Force East StratCom für „aktive strategische Kommunikation“von Unionspolitikern, um Destabilisierungsversuchen des - von der Kommission explizit genannten - russischen Staats entgegenzuwirken. Die Gruppe wird positiv beurteilt, aber die Information der Öffentlichkeit über ihre Leistungen ist nur lückenhaft und sie ist drastisch unterfinanziert. In seinen Dokumenten „drängt“ der EWSA die Kommission, die Mitgliedstaaten darauf hinzuweisen, dass ihre nationalen Institutionen und Gesetze zur Informationssicherheit unterentwickelt sind und das rechtliche Umfeld veraltet

und den Ausschuss der Regionen, Bericht über die Umsetzung des Aktionsplans gegen Desinformation v. 14.6.2019, JOIN / 2019/12 final, S.12 ist, sodass die Aufsichtsbehörden nicht in der Lage sind, Kanäle für Desinformationsaktivitäten zu kontrollieren. Ein einsamer Primus in dieser Hinsicht ist Estland, wo eine spezialisierte Regierungsverwaltung innerhalb von Stunden auf online auftauchende feindliche Desinformationen reagieren kann.

Die europäische Diagnose ist also ziemlich niederschmetternd - unterentwickelte Institutionen, ein veraltetes rechtliches Umfeld, ein deutlicher Mangel an nationalen Langzeitstrategien gegen Desinformation und ein fehlender Schutz für Bevölkerungsgruppen, die am anfälligsten für externe Desinformationskampagnen sind. Dies steht nach Ansicht des EWSA in krassem Gegensatz zu Russland, welches Desinformation sogar als eine wichtige legitime militärische Strategie behandelt wird. Die Mitgliedstaaten sind dem Microtargeting, das als Teil einer solchen Strategie eingesetzt wird, praktisch schutzlos ausgeliefert. ${ }^{3}$ Erst im Jahre 2020 konnte man die ersten ernsthaften Zeichen des Willens zur Koordinierung einer effizienten, wenn auch unterfinanzierten Gegenaktion der EU feststellen. ${ }^{4}$ Dabei unterscheiden die europäischen Dokumente klar zwischen den altbekannten Fake News (u. a. Propaganda, Hassreden, Lügen) und der Desinformation, die definiert wird als „nachweislich falsche oder irreführende Information, die mit dem Ziel des wirtschaftlichen Gewinns oder der vorsätzlichen Täuschung der Öffentlichkeit konzipiert, vorgelegt und verbreitet wird, demokratischen Prozessen Schaden zufügen und Wahlen beeinflussen kann und eine ernsthafte Bedrohung für die Gesellschaft darstellt. ${ }^{\text {“5 }} \mathrm{Da}$ bei tauchen viele neue Begriffe auf: digitale Selbstverteidigung, Desinformationskampagne, Cyberangriff, Cyberpandemie. Desinformationskette, Informationsinfrastruktur (Internet-Ökosystem) - die jedoch alle die zu späten europäischen Reaktionen auf die Bedrohungen aufzeigen ${ }^{6}$. Es sei darauf hingewiesen, dass die EWSA-Dokumente die These von Andrew Keen enthalten, dass das so genannte Businessmodell des 21. Jahrhunderts - das darauf beruht, dass man nicht mit Geld, sondern mit der Privatsphäre für die Leistungen zahlt - die Privatsphäre zerstört und eine historische, beispiellose Bedrohung für Europas Zukunft darstellt. Er betont, dass die Grundlagen der Demokratie in der EU - sichere und belastbare Wahlprozesse - bedroht sind, und macht deutlich, dass diese Sicherheit und Belastbarkeit nicht mehr gewährleistet werden kann. Bei den Wahlen der letzten Jahre wurden in 18 Ländern Desinformationen und Manipulationen aufgedeckt - ein deutlicher Hinweis auf das Ausmaß der Bedrohung.

3 Gemeinsame Mitteilung an das Europäische Parlament, den Europäischen Rat, den Rat, den Europäischen Wirtschafts- und Sozialausschuss und den Ausschuss der Regionen: Bericht über die Umsetzung des Aktionsplans gegen Desinformation (JOIN(2019) 12 final). Vgl. https://newsroom.fb.com/news/ https:// www.isdglobal.org/isd-publications/

interim-briefing-propaganda-and-digital-campaigning-in-the-eu-elections/

4 Mitteilung der Kommission an das Europäische Parlament, den Rat, den Europäischen Wirtschafts- und Sozialausschuss und den Ausschuss der Regionen: Digitaler Kompass 2030: der europäische Weg in die digitale Dekade, $\operatorname{COM}(2021) 118$ final, S. 4. Es ist symptomatisch, dass die Kommission auch im Jahre 2021 die Verstärkung der Auswirkungen der Desinformation auf unsere demokratischen Gesellschaften diagnostiziert.

5 Ibid., S. 185; vgl. COM(2018) 286, S. 4, auch C. Jack. Lexicon of Lies: Terms for Problematic Information. Raport, Data\&Society, online access: https://datasociety. net/wp-content/uploads/2017/08/DataAndSociety_LexiconofLies.pdf, 2017, S. 3.

6 A. Gryszczyńska, G. Szpor (eds), Internet. Cyberpandemia. Cyberpandemic. Warszawa 2020. Vgl. a. Mitteilung der Kommission an das Europäische Parlament, den Rat, den Europäischen Wirtschafts- und Sozialausschuss und den Ausschuss der Regionen: Digitaler Kompass 2030 - der europäische Weg in die digitale Dekade $\operatorname{COM}(2021) 118$ final, S. 2. 
Ein Teil der Lösung könnte in der Zukunft die Einführung einer neuen Kategorie der Media-Worker sein, die man als unparteiische Prüfer von Informationen bezeichnen könnte. Traditionell wurde diese Rolle von freien Medien erfüllt, die sich um die Qualität der Nachrichten kümmerten - einschließlich des öffentlichen Rundfunks und Fernsehens mit hohen Standards, für die oft die britische $\mathrm{BBC}$ als Beispiel herangezogen wurde -, inzwischen aber ihre wirtschaftliche Unabhängigkeit verlieren. In Ländern mit schwindender Demokratie werden der öffentliche Rundfunk und das Fernsehen zunehmend als eine mächtige Quelle der Desinformation statt der Information angesehen. Welche Lösung sieht die Europäische Kommission hier, auch im Zusammenhang mit COVID-19? Neben der Bereitstellung und Verbreitung von verlässlichen Inhalten und der Erhöhung der „,Resistenz" gegen Desinformation heißt es: „Wenn solche Verhaltensweisen rechtswidrig sind, sollten die zuständigen Behörden im Einklang mit den geltenden Rechtsnormen dagegen vorgehen "7. In solchen Ländern nimmt man dies eher als ein Zeugnis der Hilflosigkeit. Wenn ein Teil der Desinformation direkt von den staatlichen Medien ausgeht, kann man auch kaum auf eine effektive Strafverfolgung hoffen.

$\mathrm{Zu}$ den konkreten, positiven Errungenschaften der EU zählt die Einrichtung eines Frühwarnsystems für Desinformationsexperten im März 2019, das allen Regierungen der Mitgliedstaaten zur Verfügung steht. Ein weiteres Element ist die 2018 ergangene Aufforderung an Online-Plattformen, einen Selbstregulierungskodex zur Bekämpfung von Desinformation zu implementieren ${ }^{8}$. Trotz einiger Maßnahmen, die von digitalen Plattformen ergriffen wurden, ist es jedoch schwierig, hier von einem Erfolg zu sprechen, da die europäischen Gremien und Mitgliedstaaten eindeutig zu strengeren, absolut verbindlichen Regelungen tendieren. ${ }^{9}$

\section{Digitale Transformation in der EU}

Die EU sieht generell die digitale Transformation schon lange nicht mehr als Bedrohung, sondern als Quelle des Wohlstands. Wenn jedoch der Wettlauf gegen die Zeit verloren geht und die Desinformation, die Teil dieser Transformation ist, zum Zerfall der EU führt, wie es der Fall des Brexit befürchten lässt, werden große Programme sinnlos. Es kann argumentiert werden, dass in Ländern mit abnehmender Demokratie und Dezentralisierung nur starke Formen von EU-Rechtsmaßnahmen mit direkter Bindungskraft, wie z. B. Verordnungen, Ergebnisse bringen können.

Bislang konnte mit hoher Sicherheit festgestellt werden, dass mit dem Wandel gesellschaftlicher Bedürfnisse und Stimmungen ein periodischer Wechsel der Machteliten zwischen rechts und links bzw. in die Mitte stattfindet. Der Wechsel der Regierungen und ihrer Programme ermöglichte es dem Staat, sich stetig zu erneuern und innovativ auf neue Herausforderungen zu reagieren. Dies war u. a. die Stärke der Innovation und Kreativität in Euro-

7 Gemeinsame Mitteilung an das Europäische Parlament, den Europäischen Rat, den Rat, den Europäischen Wirtschafts- und Sozialausschuss und den Ausschuss der Regionen, JOIN(2020) 8 final, S. 4.

8 O. Pollicino, E. Bietti, Truth and Deception across the Atlantic: A Roadmap of Disinformation in the US and Europe, Italian Journal of Public Law 11, no. 1 (2019): 76-79, 84-85. Vgl. a. J. Pielemeier, “Disentangling Disinformation: What Makes Regulating Disinformation So Difficult?", Utah Law Review 2020, no. 4 (2020): 925-928.

9 J. Pielemeier, ibid., S. 931-933; T. Nagasako, Global disinformation campaigns and legal challenges. Int. Cybersecur. Law Rev. 1, 135 (2020), https://doi. org/10.1365/s43439-020-00010-7. pa und den USA. Wird dieser Wandel durch eine interne oder externe Desinformationskampagne künstlich blockiert, wird auch dieser fast natürliche Erneuerungsprozess unterbrochen. Wie die Kommission in ihrem Dokument vom 11. April 2021 feststellt, betreiben ausländische Akteure (einschließlich Russland und China, die in dem Dokument explizit genannt werden) Desinformationskampagnen (in diesem Fall rund um COVID 19), „(...) mit dem Ziel, die demokratische Debatte zu untergraben, die gesellschaftliche Polarisierung zu vertiefen und ihr eigenes Image zu verbessern ${ }^{\star 10}$. Auch das ist ein interessanter Aspekt, gerade wenn es um die soziale Polarisierung geht. Die Polarisierung unterliegt, wie auch der Regierungswechsel, periodischen Veränderungen. Die sozialen Spannungen sind manchmal stärker oder schwächer, je nach der wirtschaftlichen Situation und den aktuellen Bedrohungen, aber durch die Desinformationskampagne kommt es zu einer künstlichen Eskalation der Spannungen, die sich zerstörerisch auf die demokratische Legitimität der öffentlichen Verwaltung und der Sozialpartnerschaft auswirkt. Das Hauptopfer einer solchen künstlichen Eskalation der Spannungen ist - wie Ivan Krastev betonte - das soziale Vertrauen. Natürlich wird die Demokratie nicht nur aus diesem Grund zu einer Demokratie der Misstrauischen. ${ }^{11}$ Es war Krastev, der als erster auf die Illusion der Transparenz hinwies, d. h. auf den Defekt des "nackten“ Rechts auf Information statt des Rechts auf zuverlässige und wahre Information.

Wie in der Literatur angedeutet, führt die ständige Verschärfung der sozialen Polarisierung zu einer „digitalen Kluft“ und eliminiert die enormen Vorteile, die durch die digitale Infrastruktur erreicht werden können, insbesondere im Bereich der Gesundheitsversorgung, der ökologischen Lösungen, der beruflichen Teilhabe älterer Menschen und der Entwicklung der Telemedizin.

\section{Das spezifische Wesen von Desinformation}

Die Auswirkungen der Desinformation auf die Organisation und das Funktionieren des Staatsapparates, ihr Einfluss auf die öffentliche Verwaltung, sowohl auf die behördliche als auch auf die versorgende und die infrastrukturelle Verwaltung, werden nur unzureichend erkannt. Zum Beispiel verleitet Desinformation in der Gesundheitsversorgung unter COVID-19-Bedingungen Menschen dazu, offizielle Gesundheitsratschläge zu ignorieren und sich auf riskante Verhaltensweisen einzulassen. Die Vielzahl solcher Reaktionen wird in den Medien mit mangelnder Informiertheit in Verbindung gebracht. Selten wird in Betracht gezogen, dass dies das Ergebnis von organisierten, externen Desinformationskampagnen sein könnte, die darauf abzielen, den Staat und die Gesellschaft zu untergraben. Was früher oft als Verschwörungstheorie galt, ist heute Gegenstand offizieller Mitteilungen der Europäischen Kommission. ${ }^{12}$

\footnotetext{
10 T. Nagasako, ibid., S. 125-136.

11 Vgl. I. Krastev: Demokracja nieufnych. Warszawa 2013, S. 28-34.

12 Gemeinsame Mitteilung an das Europäische Parlament, den Europäischen Rat, den Rat, den Europäischen Wirtschafts- und Sozialausschuss und den Ausschuss der Regionen: Bekämpfung von Desinformation im Zusammenhang mit covid-19 - Fakten statt Fiktion, 10. Juni 2020 Nr. 8, S. 5. (JOIN(2020) 8 final). Fußnote 15 und 16: Die Plattform www.EUvsDisinfo.eu hat in ihrer öffentlich zugänglichen Datenbank mehr als 500 Beispiele für kremlfreundliche Desinformation veröffentlicht, und der EAD hat in seinen öffentlichen Analyseberichten die Aktivitäten aus-
} ländischer Staaten beschrieben. Siehe beispielsweise: https://euvsdisinfo.eu/de/ 
Es scheint wichtig, den oben genannten neuen Beruf des Informationsverifizierers, der vom Beruf des Journalisten oder Datenanalysten $\mathrm{zu}$ unterscheiden ist, $\mathrm{zu}$ institutionalisieren und $\mathrm{zu}$ finanzieren. Ein interessantes Beispiel aus Polen ist das des Ombudsmann-Preisträgers Michał Rogalski, der im Alter von 19 Jahren eine verlässliche, allgemein zugängliche und weit verbreitete statistische Datenbank über COVID-19-Fälle aufgebaut hatte. Diese Datenbank gewann schnell enormes öffentliches Vertrauen und trug dazu bei, das Niveau der öffentlichen Debatte zu erhöhen. ${ }^{13}$ Es war ein ehrenamtliches, freiwilliges bürgerschaftliches Engagement für das Gemeinwohl, bei dem es sich um eine Aktivität handelte, die kostenfrei, als Teil eines gemeinsamen Kampfes gegen eine gefährliche Pandemie, zur Verfügung gestellt wurde. Dieses bürgerschaftliche Engagement ist durch das Bestreben gekennzeichnet, eine Gemeinschaft um das Projekt herum aufzubauen. Michał Rogalski hat nicht allein gehandelt, sondern Menschen unterschiedlichen Alters und unterschiedlicher Berufe überzeugt, zusammenzuarbeiten. Diese Aktivität erwies sich als so nützlich, dass korrekte und klar aggregierte Informationen nicht nur von den Medien und sozialen Aktivisten, sondern auch von Universitäten zu Forschungszwecken, von der Kommunalverwaltung und der öffentlichen Verwaltung verwendet wurden. Erst als diese Tatsache an die Öffentlichkeit gelangte, wurde ihm ein Teil der Informationen vorenthalten und seine Berichterstattung von den Sanitätsstationen vor Ort geändert. Es gibt aber keine Belege dafür, dass diese Zentralisierung eine Verbesserung der Informationsbasis und der Transparenz der öffentlichen Verwaltungstätigkeit bewirkt hätte.

Zusammenfassend: Die öffentliche Reaktion auf die Pandemie bestätigt eindrucksvoll die Richtigkeit von Angelo Codevillas Ansicht, dass Desinformation „fast nie versucht, die Wahrheit zu verbergen. Vielmehr besteht sie darin, ein ausreichend breites Feld an intellektuellen Möglichkeiten zu schaffen, damit die eigenen Präferenzen des Gegners in seinem Kopf in den Vordergrund treten können. Das Wesen der Desinformation besteht darin, dem Gegner ein Argument zu liefern, damit er glaubt, was er glauben will. Nichts in der Welt wird einen Menschen von dem überzeugen, was er nicht glauben will, während jeder Vorwand ausreicht, um ihn dazu zu bringen, auch viele Beweise zu ignorieren, die mit den Schlussfolgerungen, die er ziehen möchte, in Konflikt stehen. ${ }^{\text {"14 }}$ Diese Aussage aus den 1990er Jahren verbindet Desinformation mit dem in neueren Publikationen beliebten Begriff Post-Truth, der Umstände bezeichnet oder definiert, in denen Fakten die öffentliche Meinung weniger prägen als Emotionen und persönliche Überzeugungen. ${ }^{15}$

ead-sonderbericht-update-kurzbewertung-der-narrative-und-desinformation-zurcovid-19-pandemie-aktualisierung-23-april-bis-18-mai/.

13 https://www.rpo.gov.pl/pl/content/michal-rogalski-uroczystosc-wreczenia-nagrody-rpo-im-pawla-wlodkowica, Laudacja Prof. Ireny Lipowicz.

14 A. Codevilla, Informing Statecraft. Intelligence for the New Century, The Free Press, New York 1992, S.334.

15 "(...) relating to or denoting circumstances in which objective facts are less influential in shaping public opinion than appeals to emotion and personal belief", https://en.oxforddictionaries.com. Vgl. Lejla Turcilo and Mladen Obrenovic, A Companion to Democracy \#3. Misinformation, Disinformation, Malinformation: Causes, Trends, and Their Influence on Democracy, A Publication of Heinrich Böll Foundation, 2020. S. 17-22.

\section{Perspektiven}

Bei der rechtlichen Betrachtung von Desinformation als Bedrohung, die durch die digitale Transformation entstanden ist, kann es hilfreich sein, Daten und Nachrichten [Messages] von Informationen zu unterscheiden und sich dabei auf die Informationstheorie zu berufen, die als Maß für die Informationsmenge die Menge an Ungewissheit [Entropie] nimmt, die durch das Eintreten eines Ereignisses (Empfang einer Nachricht) ${ }^{16}$ beseitigt worden ist. Durch die Anhäufung von Datenressourcen kann die Reduzierung von Unsicherheit sogar erschwert werden. Es sollte davon ausgegangen werden, dass nur die Nachricht [Ankündigung], die Unsicherheit reduziert, also wahr ist, eine Information ist und falsche Nachrichten, die keinen Informationswert haben, nicht als Information $\mathrm{zu}$ bezeichnen sind. ${ }^{17}$

Unter Berücksichtigung dieses Postulats kann Desinformation als eine Kombination von Informationen und Falschnachrichten erklärt werden, die so übermittelt werden, dass der Adressat irregeführt und zu einem erwartungsgemäßen und für den Gegner/ Wettbewerber vorteilhaften Verhalten veranlasst wird. Eine Desinformationsaktion besteht in der Regel darin, ein ganzes Konglomerat von Informationen zu übermitteln, an das eine einzige Falschmeldung angehängt wird - entscheidend für die Herbeiführung der angenommenen Wirkung. Eine Desinformationsaktion kann auch auf der Grundlage von Informationen durchgeführt werden, die jedoch bewusst so vermittelt werden, dass ein Adressat sie als falsch wahrnimmt ${ }^{18}$.

Wahrhaftigkeit als immanentes Attribut von Information zu behandeln, könnte die Lösung vieler aktueller Probleme erleichtern und würde lediglich erfordern, den Begriff „Falschinformation“ durch „Falschmeldung“ (Fake News) zu ersetzen. Die Valorisierung von Informationen würde auch unter den Bedingungen der digitalen Transformation die Stabilität der rechtlichen Grundlagen der Informationsfreiheit sichern und gleichzeitig Desinformation vom rechtlichen Schutz ausschließen.

Hervorzuheben ist auch, dass (wie der Ausschuss der Regionen als Organ der Europäischen Union betont) eine wirksame Bekämpfung von Desinformation in erster Linie intensive Maßnahmen auf lokaler Ebene erfordert, einschließlich der Stärkung der lokalen Medien. Auch die von der Kommission erstellten Berichte über die Umsetzung der Strategie gegen Desinformation bestätigen die Richtigkeit dieses Ansatzes; Desinformationskampagnen, auch von außen, sind auf lokaler Ebene leichter zu verbergen und werden dort intensiviert. In diesem Zusammenhang erscheint die vom Ausschuss der Regionen vorgeschlagene Strategie besonders gerechtfertigt. Auf lokaler Ebene sollten neue Netzwerke von Informationsverifikatoren mit europäischen Mitteln kofinanziert werden. ${ }^{19}$

16 S. Mynarski, Elementy teorii systemów i cybernetyki, 1979, S. 155.

17 G. Szpor, Jawność i jej ograniczenia . T.1 Idee i pojęcia, Warszawa 2016, S. 250

18 Vgl. T. Aleksandrowicz, Podstawy walki informacyjnej, Warszawa 2016, S. 83 i 84; O. Pollicino, E. Bietti, Truth and Deception across the Atlantic: A Roadmap of Disinformation in the US and Europe, Italian Journal of Public Law 11, no. 1 (2019): p. 49; J. Pielemeier, Disentangling Disinformation: What Makes Regulating Disinformation So Difficult?, 2020 UTAH L. REV. 917 (2020), str. 920; C. Jack, Lexicon of Lies: Terms for Problematic Information. Report, Data\&Society, S. 14-15, online access: https://datasociety.net/wp-content/uploads/2017/08/ DataAndSociety_LexiconofLies.pdf, 2017.

19 Gemeinsame Mitteilung an das Europäische Parlament, den Europäischen Rat, den Rat, den Europäischen Wirtschafts- und Sozialausschuss und den Ausschuss der Regionen. Bericht über die Umsetzung des Aktionsplans gegen Desinformation. JOIN(2019) 12 final, S. 5. 